\title{
Acknowledgement to Reviewers of Separations in 2018
}

\author{
Separations Editorial Office
}

MDPI, St. Alban-Anlage 66, 4052 Basel, Switzerland

Published: 18 January 2019

Rigorous peer-review is the corner-stone of high-quality academic publishing. The editorial team greatly appreciates the reviewers who contributed their knowledge and expertise to the journal's editorial process over the past 12 months. In 2018, a total of 56 papers were published in the journal, with a median time to first decision of 23 days and a median time to publication of 62.5 days. The editors would like to express their sincere gratitude to the following reviewers for their cooperation and dedication in 2018:

$\begin{array}{ll}\text { Abete, Maria Cesarina } & \text { Galván-D'Alessandro, Leandro } \\ \text { Alladio, Eugenio } & \text { Gheju, Marius } \\ \text { Amigo-Benavent, Miryam } & \text { Gilchrist, Elizabeth } \\ \text { Anticó, Enriqueta } & \text { Gonçalves, Virginia } \\ \text { Baeza Baeza, Juan José } & \text { Gong, Maojun } \\ \text { Bakry, Rania } & \text { Griffiths, Peter } \\ \text { Barreca, Salvatore } & \text { Grinias, James P. } \\ \text { Berhow, Mark } & \text { Gumieniczek, Anna } \\ \text { Blanchard, Peter E.R. } & \text { Gunschera, Jan } \\ \text { Bonikowski, Radosław } & \text { Heberger, Karoly } \\ \text { Bruno, Thomas J. } & \text { Herráez-Hernández, Rosa } \\ \text { Burgio, Lucia } & \text { Hlozek, Tomas } \\ \text { Campíns-Falcó, Pilar } & \text { Hopfer, Helene } \\ \text { Carrero, Jose Antonio } & \text { Iadarola, Paolo } \\ \text { Chen, Hsin-Chun } & \text { Ifelebuegu, Augustine } \\ \text { Cheng, Keding } & \text { Jandera, Pavel } \\ \text { Coldea, Teodora } & \text { Järvenpää, Eila P. } \\ \text { Cologna, Stephanie } & \text { Jaumot, Joaquim } \\ \text { Cosma, Pinalysa } & \text { Kalíková, Kvĕta } \\ \text { Dal Bello, Federica } & \text { Kataoka, Hiroyuki } \\ \text { Del Hoyo, Carmen M. } & \text { Kim, Daehwan } \\ \text { Destandeau, Emilie } & \text { Klarskov, Klaus } \\ \text { Devasurendra, Amila } & \text { Koel, Mihkel } \\ \text { Dichiara, Anthony } & \text { Kopel, Pavel } \\ \text { Dinica, Rodica Mihaela } & \text { Korzeniowska, Margorzata } \\ \text { Dolowy, Malgorzata } & \text { Koziel, Jacek A. } \\ \text { Douvris, Christos } & \text { Kozliak, Evguenii I. } \\ \text { Dunphy, Michael J. } & \text { Kulbacka, Julita } \\ \text { Escudero, Carlos } & \text { Kusch, Peter } \\ \text { Esteve-Romero, Josep } & \text { Laborda, Francisco } \\ \text { Falconer, James } & \text { Lantes, Oscar } \\ \text { Galbács, Gábor } & \text { Li, Ailin } \\ & \end{array}$


Liao, Shu-Chuan

Locatelli, Marcello

Lopez-Linares, Francisco

Lucena, Rafael

Luna, Carlos

Luo, Wentai

Maage, Amund

Madrid, Yolanda

Marta, Ferreiro-González

Martín-Alberca, Carlos

Martins, Rui C.

Mashima, Ryuichi

Matikonda, Siddharth

Miękus, Natalia

Mirna, Habuda-Stanic

Mitulovic, Goran

Miyazato, Hironari

Mudalige, Thilak K.

Nam, Hyungseok

Nasiri, Rasoul

Nic Daeid, Niamh

Nischang, Ivo

Nthunya, Lebea N.

Oliveira, Hugo M.

Onchoke, Kefa

Palma, Miguel

Panderi, Irene

Parpinello, Giuseppina

Pazourek, Jiri

Perepelyuk, Maryna

Perestrelo, Rosa

Perrault, Katelynn A.

Philippe, Allan

Picca, Rosaria Anna

Pintado-Herrera, Marina

Piriou, Yannick

Pohl, Pawel

Pollmann, Katrin

Pozo, Óscar J.

Prochaska, Charikleia

Prosen, Helena
Protti, Michele

Puton, Jarosław

Ramil, María

Ramos, Daniel

Roby, Mohamed Hussein Hamdy

Rodriguez, Pilar

Rosenberg, Erwin

Roy, Sagar

Ruiz-Aceituno, Laura

Saiano, Filippo

Sarti, Elena

Schepdael, Ann Van

Schwartz, Andrew J.

Shin, Jae Sup

Skalicka-Woźniak, Krystyna

Snow, Nicholas

Stefanuto, Pierre-Hugues

Sterba, Johannes H.

Stürup, Stefan

Szumny, Antoni Jacek

Takahashi, Katsuyuki

Torres-Padrón, M. Esther

Trögl, Josef

Troise, Antonio Dario

Tuzimski, Tomasz

Tyśkiewicz, Katarzyna

Urieta, José

Valko, Klara

Vázquez-Tato, M. Pilar

Verzera, Antonella

Viapiana, Agnieszka

Vila Verde, Giuliana M.

Wahab, M. Farooq

Walsh, Marie

Wätzig, Hermann

Willerth, Stephanie M.

Witkiewicz, Zygfryd

Woźniak, Łukasz

Yoza, Brandon A.

Zajickova, Zuzana

Zielińska-Pisklak, Monika A.

(C) 2019 by the authors. Licensee MDPI, Basel, Switzerland. This article is an open access article distributed under the terms and conditions of the Creative Commons Attribution (CC BY) license (http://creativecommons.org/licenses/by/4.0/). 